\title{
CONVEX FUNCTIONS AND HARMONIC MAPS
}

\author{
WILLIAM B. GORDON
}

\begin{abstract}
A subset $D$ of a riemannian manifold $Y$ is said to be convex supporting if every compact subset of $D$ has a $Y$-open neighborhood which supports a strictly convex function. The image of a harmonic map $f$ from a compact manifold $X$ to $Y$ cannot be contained in any convex supporting subset of $Y$ uniess $f$ is constant. Also, if $Y$ has a convex supporting covering space and $\pi_{1}(X)$ is finite then every harmonic map from $X$ to $Y$ is necessarily constant. Examples of convex supporting domains and manifolds are given.
\end{abstract}

1. Notation. $X$ and $Y$ will always denote $C^{\infty}$ riemannian manifolds and it will always be assumed that $X$ is compact. Greek (Latin) letters will be used for objects attached to $X(Y)$. Local coordinates will be written $x=\left(x^{a}\right), y=\left(y^{i}\right)$, and the corresponding metric tensors and Christoffel symbols will be denoted by $g=\left(g_{\alpha \beta}\right), h=\left(h_{i j}\right), \Delta_{\beta \gamma}^{\alpha}, \Gamma_{j k}^{i}$. Indices of tensors will be raised and lowered in the usual fashion, and we shail always use the tensor summation convention. If $F$ is a $C^{2}$ function on $Y$ we write $F_{i}$ for $\partial F / \partial y^{i}$ and $F_{i j}$ for $\partial^{2} F / \partial y^{i} \partial y^{j}-\Gamma_{i j}^{k} F_{k}$. $F$ is said to be strictly convex on an open subset $U$ of $Y$ if the Hessian form $\left(F_{i j}\right)$ is strictly positive at every point of $U$. Let $f: X \rightarrow Y$ be a $C^{2}$ map which is locally given by $y=y(x)$. We set

$$
y_{\alpha}^{i}=\frac{\partial y^{i}}{\partial x^{\alpha}} ; \quad y_{\alpha \beta}^{i}=\frac{\partial^{2} y^{i}}{\partial x^{\alpha} \partial x^{\beta}}-\Delta_{\alpha \beta}^{\gamma} y_{\gamma}^{i}+\Gamma_{j k}^{i} y_{\alpha}^{j} y_{,}^{k} .
$$

Then $y_{\alpha}^{i}$ and $y_{\alpha \beta}^{i}$ transform like tensors under the coordinate transformations $x \rightarrow \bar{x}, y \rightarrow \bar{y}$. We recall that $f$ is said to be harmonic iff

$$
g^{\alpha \beta} y_{\alpha \beta}^{i}=0 \quad(i=1,2, \cdots, \operatorname{dim}(Y)) .
$$

For motivation and some results in the theory of harmonic maps see [2], [4], [5]. Equations (2) are the Euler-Lagrange equations for the "energy" functional $E(f)=\int_{X} g^{\alpha \beta} h_{i j} y_{\alpha}^{i} y_{\beta}^{j} \sqrt{ } g d x$.

2. A subset $A$ of $Y$ will be said to be convex supporting iff every compact subset of $A$ has a $Y$-open neighborhood on which there is defined a strictiy convex function $F$. Note that $F$ need not be globally defined. (Examples of

Received by the editors September 3, 1970.

AMS 1970 subject classifications. Primary 49F99; Secondary 58C05.

Key words and phrases. Harmonic map, convex function.

(c) American Mathematical Society 1972 
convex supporting domains and manifolds are given in §4.) Our main results are summarized in the following theorem.

THEOREM. Let $X$ and $Y$ be $C^{\infty}$ riemannian manifolds with $X$ compact and connected.

(A) The image of any harmonic map $f: X \rightarrow Y$ cannot be contained in any convex supporting subset of $Y$ unless $f=$ constant. Hence, any harmonic map from $X$ to $Y$ is necessarily constant if $Y$ is convex supporting.

(B) If $\pi_{1}(X)$ is finite (i.e., the universal covering space of $X$ is compact) and $Y$ has a covering space which is convex supporting with respect to the lifted metric of $Y$, then every harmonic map from $X$ to $Y$ is necessarily constant.

EXAmPle. Bishop and O'Neill [1] have recently shown that the existence of a globally defined convex function on a manifold imposes strong conditions on the structure of the manifold, especially manifolds of nonpositive sectional curvature. Among their results, we note the following two:

(a) A compact manifold cannot be convex supporting.

(b) A complete simply connected manifold $M$ whose riemannian sectional curvatures are all $\leqq 0$ supports a convex function; viz., if $p_{0}$ is a fixed point on $M$ and $F(p)=$ squared geodesic distance from $p_{0}$ to $p$, then $F$ is strictly convex. Hence the universal covering space of a complete riemannian manifold with nonpositive sectional curvatures is convex supporting.

Now according to a well-known theorem of Myers, if the Ricci curvature form of a manifold $X$ is strictly positive definite, then $X$ and its universal covering space are compact (see e.g. [6, p. 105]). Hence we obtain the known result that every harmonic map from $X$ to $Y$ is necessarily constant if the Ricci curvature of $X$ is positive definite and the riemannian curvatures of $Y$ are all nonpositive. Note however that this fact does not depend on the riemannian structure of $X$. E.g., if the standard metric on $S^{n}(n>1)$ is replaced with an arbitrary metric, it still remains true that every harmonic map from $S^{n}$ to a manifold with nonpositive riemannian curvatures is necessarily constant. (Cf. the corollary on p. 124 of [2].)

3. The proof. Without loss of generality we can assume that $X$ is orientable, since otherwise we can apply the following arguments to the orientable cover of $X$.

For (A), let $F$ be a function which is strictly convex in a neighborhood of $f(X)$, and let $G=F \circ f$. Using the notation of $\S 1$, we get $G_{\alpha \beta}=F_{i} y_{\alpha \beta}^{i}+F_{i j} y_{\alpha}^{i} y_{\beta}^{j}$. Multiplying by $g^{\alpha \beta}$ using (2) and integrating we get $\int_{X} g^{\alpha \beta} G_{\alpha \beta} \sqrt{ } g d x=$ $\int_{X} g^{\alpha \beta} F_{i j} y_{x}^{i} y_{\beta}^{j} \sqrt{ } g d x$. The left-hand side vanishes because the integrand is 
the Laplacian of $G$, and, from convexity, the right-hand side can only vanish if $y_{\alpha}^{i} \equiv 0$.

To prove (B) let $\pi: \tilde{X} \rightarrow X$ be a universal covering of $X, \rho: \tilde{Y} \rightarrow Y$ a covering for which $\tilde{Y}$ is convex supporting with respect to the metric $\rho * h$. Let $f: X \rightarrow Y$ be harmonic and set $\hat{f}_{1}=f \circ \pi$. Then $f_{1}$ is harmonic. But $\tilde{X}$ is simply connected, so that from general principles (see e.g. $\left[7\right.$, p. 76]) $f_{\text {: }}$ lifts to a map $f_{2}$ from $\tilde{X}$ to $\tilde{Y}$ which satisties $\rho \circ f_{2}=f_{1}$. Using the fact that the local inverses of $\rho$ are isometries it is easy to show that $f_{2}$ is harmonic, and from (A) it follows that $f_{2}$ and therefore $f$ is constant.

4. Examples and applications. (i) Every point $p$ has a convex supporting neighborhood. (Proof. For a coordinate system $y$ centered at $p$, set $F(y)=\Sigma\left(y^{i}\right)^{2}$.) Hence from (A), we can say that the image of a harmonic map cannot become too small without collapsing to a point.

(ii) A closed geodesic or $Y$ is a harmonic map from the circle $S^{1}$ to $Y$. Hence a convex supporting manifold cannot contain a closed geodesic (cf. [1, p. 5]). Hence in particular, the hyperboloid of revolution $x^{2}+y^{2}=$ $1+z^{2}$ is not convex supporting. However the upper and lower halves of the hyperboloid are convex supporting: take $F(p)=$ squared distance from axis of rotation.

(iii) The surface of revolution $x^{2}+y^{2}=e^{-z}$ is convex supporting (with $F^{\prime}$ as above). These last two examples are diffeomorphic and have negative curvatures.

(iv) The paraboloid of revolution $x^{2}+y^{2}=z^{2}$ is an example of a convex supporting manifold with positive curvature ( $F$ as before).

(v) Let $p$ be a point on the circle $S^{1}$. Then $S^{1}-\{p\}$ is a convex supporting domain. Hence any harmonic map from a compact manifold to $S^{1}$ is either constant or surjective.

(vi) On the torus $T^{2}$ endowed with the flat metric, the open set $D$ obtained by removing two transverse circles is convex supporting. For if the torus is constructed in the usual manner by glueing the edges of a rectangle together, the transverse circles can be identified with an adjacent pair of edges, and it is easy to see that any closed subset of the rectangle which does not intersect any edge is convex supporting.

(vii) Every open convex supporting subset of a riemannian manifold is contained in a maximal open convex supporting set; i.e., one which is not properly contained in any other open convex supporting set. (Similarly, every connected open convex supporting set is contained in a maximal connected open convex supporting set.) Proof. Use Zorn's lemma. Let $\Gamma$ be a family of open convex supporting subsets which is linearly ordered by the inclusion relation. Then $U \Gamma$ is convex supporting since any compact subset of $U \Gamma$ is contained in one of the members of $\Gamma$. 
The convex supporting domain $D$ described in (vi) is maximal because any larger open subset of $D$ contains a closed geodesic. Also, from (i) it follows that the complement of a maximal open convex supporting subset is nowhere dense.

(viii) Consider the ordinary 2-sphere $S^{2}$ equipped with the standard riemannian metric. Then each of the open hemispheres obtained by removing a great circle is convex supporting (take $F(p)=$ square of chord joining $p$ to the nearest pole) but the union of the two hemispheres is not a maximal open convex supporting open set and neither hemisphere is a maximal connected open convex supporting set. In order to obtain a maximal open convex supporting domain (which happens to be connected) it suffices to remove half of a great circle; i.e., an arc $\gamma$ joining two poles. (The verification of this fact is somewhat tedious. Let $(\theta, \varphi)$ be standard spherical coordinates with $\theta=$ polar angle. We allow $\theta$ and $\varphi$ to run from 0 to $\pi$ and from 0 to $2 \pi$ respectively. Let $\gamma$ be the half-circle at $\varphi=0$ which joins the poles. Let $D$ be $S^{2}$ with $\gamma$ removed, and let $K$ be a compact subset of $D$. If we set $F(\theta, \varphi)=\{-1+(1-1 / n) \exp (\varphi / n)\} \sin \theta$, it turns out that the Hessian matrix of $F$ is diagonal and that the diagonal entries are positive over a range of values of $(\theta, \varphi)$ sufficient to cover $K$ provided that $n$ is sufficiently large. The value of $n$ depends on $K$.)

(ix) Finally, we mention an application to dynamics. Let $F$ be a function which is defined and strictly convex on some domain $D$ and let $\gamma=\gamma(t)$ be a geodesic segment in $D$. Then $F \circ \gamma$ is a convex function on $R$. Hence it is easy to show that every (not necessarily closed) geodesic which enters a compact convex supporting domain must eventually leave the domain. Now consider the conservative dynamical system associated with a potential $V$ on a manifold $Y$. I.e., we consider the trajectories of the system

$$
D^{2} x / d t^{2}=-\nabla V
$$

As is well known every solution to (*) with total energy $H$ is a re-parametrized geodesic with respect to the Jacobi metric $\hat{h}_{i j}=(H-V) h_{i j}$ (where $h_{i j}$ is the metric tensor of $Y$ ). Hence one can construct compact domains with the property that every solution of $(*)$ with total energy $H$ which enters such a domain must leave the domain. The sizes of these domains depend on $H$.

\section{REFERENCES}

1. R. L. Bishop and B. O'Neill, Manifolds of negative curvature, Trans. Amer. Math. Soc. 145 (1969), 1-49. MR 40 \#4891.

2. J. Eells, Jr. and J. H. Sampson, Harmonic mappings of Riemannian manifolds, Amer. J. Math. 86 (1964), 109-160. MR 29 \#1603.

3. L. P. Eisenhart, Riemannian geometry, Princeton Univ. Press, Princeton, N.J., 1926. 
4. F. B. Fuller, Harmonic mappings, Proc. Nat. Acad. Sci. U.S.A. 40 (1954), 987-991. MR 16, 284.

5. P. Hartman, On homotopic harmonic maps, Canad. J. Math. 19 (1967), 673-687. MR 35 \#4856.

6. J. Milnor, Morse theory, Ann. of Math. Studies, no. 51, Princeton Univ. Press, Princeton, N.J., 1963. MR 29 \#634.

7. E. H. Spanier, Algebraic topology, McGraw-Hill, New York, 1966. MR 35 \#1007.

Mathematics Research Center, Naval Research laboratory, Washington, D.C. 20390 